\title{
Space VLBI
}

\author{
H. Hirabayashi \\ Institute of Space and Astronautical Science, 3-1-1 Yoshinodai, \\ Sagamihara, Kanagawa 229-8510, Japan
}

\begin{abstract}
Space VLBI enables high angular resolution and high dynamic range imaging through an extension of ground-based VLBI. The TDRSS space VLBI experiments in the 1980s were followed by the first space VLBI imaging mission, VSOP, in the 1990s. The new century holds the promise of the VSOP-2 and ARISE missions, which aim for more sensitive, higher angular resolution and higher observing frequency capabilities. These missions will enable AGNs to be viewed much more clearly and will make broader science areas, including lower brightness sources, accessible. It is noted that in all space VLBI missions, international collaboration in global sense plays an important role.
\end{abstract}

\section{Historical Introduction to Space VLBI}

Radio-astronomy is most handicapped by the diffraction limited angular resolution, but is best suited for interferometry. Discussions toward improving the resolution of Very Long Baseline Interferometry (VLBI) arrays by having at least one element on a spacecraft - 'space VLBI' - commenced soon after the first successful VLBI observations were made (see, e.g., Burke 1984). The feasibility of VLBI with an orbiting element was demonstrated in a series of experiments with a Tracking and Data Relay Satellite System (TDRSS) communications satellite. Observations using the $4.9 \mathrm{~m}$ antenna of a TDRSS satellite and 2-3 ground radio telescopes at $2.3 \mathrm{GHz}$ and $15 \mathrm{GHz}$ were made between 1986 and 1988, demonstrating that the basic concepts of space VLBI worked and that sources could be detected on baselines up to two Earth diameters (Levy et al. 1986; Linfield et al. 1989; Linfield et al. 1990).

The proposed QUASAT (Schilizzi 1988) and IVS (Pilbratt 1991) missions played a significant role in focusing people's attention on the possibilities, and challenges, of space VLBI. The formerly Soviet, now Russian, RadioAstron (Kardashev and Slysh 1988; Kardashev 1997) and Japanese VSOP missions (Hirabayashi et al. 1998) were later funded, with RadioAstron targeted at high brightness source observations and VSOP aiming for a better imaging capability. Dedicated space VLBI was finally realised with the launch of the VSOP spacecraft, HALCA, in February 1997, thirty years after the first VLBI experiments by Canadian, US and British radioastronomers, and half a century after the first detection of fringes by the cliff-top interferometer in Sydney (Bolton and Stanley 1948). 
HALCA synthesizes an aperture of $30,000 \mathrm{~km}$ and carries $1.6,5$ and $22 \mathrm{GHz}$ receivers, making 1, 0.3, 0.08 mas angular resolution possible. However, the $22 \mathrm{GHz}$ sensitivity is limited because of damage experienced during launch, and scientific observations are made at 1.6 and $5 \mathrm{GHz}$. Most of the scientific targets are AGN cores, jet morphology and component motions, AGN disk absorption, GPS spectral studies, etc., with some pulsar, and $\mathrm{OH}$ maser line mapping also being done. HALCA is being operated for science observations as part of the VSOP (VLBI Space Observatory Programme) project in cooperation with many organizations and radio telescopes around the world.

VSOP-2 (ISAS) and ARISE (NASA) are missions planned for the future, and these aim for more sensitive, higher angular resolution and higher observing frequency capabilities. These missions will allow AGNs to be viewed much more clearly and broader science areas, including lower brightness or weaker sources, will become accessible.

\section{The VLBI Space Observatory Programme}

It is noted that in all space VLBI missions, international collaboration in global sense plays an important role. For this reason, a short review of the VSOP mission will be given as it is the only operating mission. The Institute of Space and Astronautical Science (ISAS) started the VSOP project in 1989, and launched HALCA into Earth orbit on 1997 February 12. HALCA's orbit has an apogee height of $21,400 \mathrm{~km}$, perigee height of $560 \mathrm{~km}$, inclination angle of $31^{\circ}$, and orbital period of 6.3 hours. The $8 \mathrm{~m}$ main reflector and the sub-reflector supporting structure were deployed in orbit. An artist's impression of the deployed satellite observing in concert with a number of ground telescopes is shown in Figure 1.

After a series of experiments as an engineering satellite - deployment of the 8 meter antenna, precise attitude control of spacecraft with a large antenna, transfer of phase reference signal, high data rate telemetry, etc. - fringes were first detected between the Usuda $64 \mathrm{~m}$ antenna and HALCA, via the Usuda $10 \mathrm{~m}$ tracking antenna, from an observation of the quasar PKS 1519-273 at $1.6 \mathrm{GHz}$ on 1997 May 7. The tapes were processed with the VSOP correlator at the National Astronomical Observatory (NAO), Mitaka. In April 1998, $22 \mathrm{GHz}$ fringes to HALCA were first detected, at the Socorro correlator, for the bursting Orion KL water maser (Kobayashi et al. 2000). HALCA has had some operational problems resulting in observing being stopped because of telemetry signal loss, attitude control loss, etc. However, as far as electric power is concerned, it seems HALCA will be able to operate for at least five years from launch.

\section{VSOP International Operation}

HALCA is commanded from the Sagamihara Operations Center at ISAS by $2.1 \mathrm{GHz}$ telemetry through the Kagoshima Space Center. For VLBI observations real-time two-way telemetry support with phase-transfer uplink at $15.3 \mathrm{GHz}$ and data downlink at $14.2 \mathrm{GHz}$ at $128 \mathrm{Mbps}$ is required, and so, five $15 \mathrm{GHz}$ band tracking stations are employed: at Usuda (Japan), operated by ISAS; Goldstone (USA), Madrid (Spain), and Tidbinbilla (Australia) operated by NASA/JPL; and Green Bank (USA), operated by NRAO. Range-rate data from 


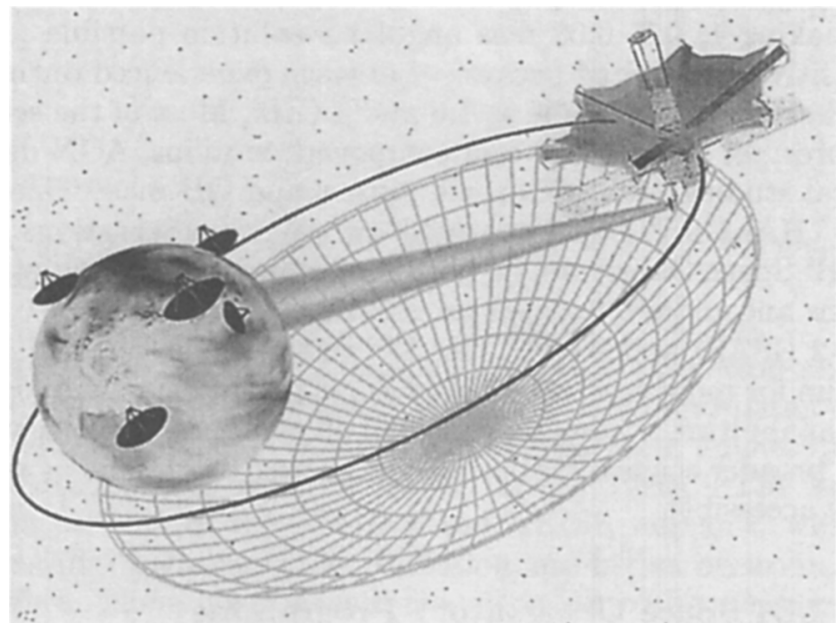

Figure 1. The HALCA satellite co-observing (via a real-time twoway link with a tracking station) with ground radio telescopes for VLBI Space Observatory Programme (VSOP) observations.

this ground telemetry network is also used for ISAS and JPL orbit determination (Hirabayashi et al. 2000a).

A number of ground radio telescopes have committed time for simultaneous observations with HALCA. The commitments of ground radio telescopes have been negotiated through the Global VLBI Working Group (GVWG), formed under URSI Commission J. Participating telescopes include those of the Very Long Baseline Array (VLBA), the European VLBI Network (EVN), the AsiaPacific Telescope, and individual telescopes.

The recording format of the VLBI data is dependent upon the ground facilities involved. Three different tape formats are used: S2, VSOPT and VLBA/MkIV. Depending on the participating ground telescopes, VSOP observations are correlated at the 10-station VSOP correlator in Mitaka, the 20station VLBA correlator in Socorro, the 6-station S2 correlator in Penticton. Experiments involving two or more formats require conversion of some tapes at Mitaka, which has two S2/VSOPT and two VLBA/VSOPT bi-directional copiers.

ISAS invited international partners to form the VSOP International Science Council (VISC) to determine and guide the science policy of the VSOP mission several years before HALCA's launch. Scientific observations are classified into two categories; the peer-reviewed General Observing Time Program and the mission-led Survey Program. The Survey Program deals with a statistical sample of nearly 300 sources, observed at $5 \mathrm{GHz}$ with $\sim 3$ ground telescopes over $\sim 6$ hours, and is described in more detail by Hirabayashi et al. (2000b). Scientific observations are being routinely undertaken at 1.6 and $5 \mathrm{GHz}$, with well over 500 observations having been made to date. 


\section{Scientific Results from the VSOP Mission}

Results of VSOP observations of AGN are reviewed by Murata et al. (2001). A good summary of the mission at the epoch of January 2000 can be seen in the VSOP Symposium Proceedings "Astrophysical Phenomena Revealed by Space VLBI". These proceedings are also available on-line from the VSOP web site, at http://www.vsop.isas.ac.jp/vsopsymp/papers. (Interesting results from VSOP observations of galactic sources - $\mathrm{OH}$ masers, pulsars and scattering phenomena - are also included.)

\section{The Future of Space VLBI}

The VSOP mission, if it were more sensitive to lower brightness sources, could do more science for gravitationally lensed objects, galactic super-luminal or subluminal sources, flare stars, late-type stars, continuum AGN cores with rotating $\mathrm{H}_{2} \mathrm{O}$ maser disks, etc. $\mathrm{H}_{2} \mathrm{O}$ maser and continuum observations at $22 \mathrm{GHz}$ were planned as one of the most important goals for the VSOP mission in terms of AGN and maser physics, but have been unable to be carried out.

In contrast to the general trend to higher observing frequency and higher resolution, there are intriguing possibilities for space VLBI at very low frequencies. At frequencies below a few tens of $\mathrm{MHz}$, the ionosphere prevents ground based observations, and so a space-based array would be required. Such an array would enable a broad range of science to be undertaken, ranging from the study of coronal mass ejections from the Sun, to the discovery of high redshift galaxies and "fossil" radio lobes (Jones et al. 2000).

There are many frontiers or possible directions for future space-VLBI; longer baselines for higher angular resolution, shorter observing wavelengths, better sensitivity, lower brightness detection limit, etc. Multiple space element interferometry for ground-space VLBI has a lot of merit, and will undoubtedly be realised at some stage in the future.

Table 1. Comparison between VSOP, VSOP-2 and ARISE

\begin{tabular}{llll}
\hline \hline & VSOP & VSOP-2 & ARISE \\
\hline Antenna Diameter & $8 \mathrm{~m}$ & $\sim 12 \mathrm{~m}$ & $25 \mathrm{~m}$ \\
Antenna Design & On-axis & Off-set parabola & Off-set parabola \\
Apogee Height $(\mathrm{km})$ & $21,400 \mathrm{~km}$ & $\sim 30,000 \mathrm{~km}$ & $40,000 \sim 100,000 \mathrm{~km}$ \\
Period & $6.3 \mathrm{hr}$ & $8.9 \mathrm{hr}$ & $13 \sim 37 \mathrm{hr}$ \\
Polarization & $\mathrm{LCP}$ & $\mathrm{LCP}$ and RCP & LCP and RCP \\
Observing Bands & $1.6,5 \mathrm{GHz}$ & $5,22,43 \mathrm{GHz}$ & $8,22,43,86 \mathrm{GHz}$ \\
Maximum Resolution & $300 \mu \mathrm{as}$ & $25 \mu \mathrm{as}$ & $20 \sim 8 \mu \mathrm{as}$ \\
Downlink bit rate & $128 \mathrm{Mbps}$ & $1 \mathrm{Gbps}$ & $4 \sim 8 \mathrm{Gbps}$ \\
System Temperature & $\sim 90 \mathrm{~K}$ & $\sim 30 \mathrm{~K}$ & $10 \sim 50 \mathrm{~K}$ \\
\hline \hline
\end{tabular}

A Japanese successor to VSOP, dubbed VSOP-2, is being considered as a near-future mission with a potential launch of 2008 (Hirabayashi et al. 2000c; 
Hirabayashi et al. 2001; see also http://www.vsop.isas.ac.jp/vsop2.html). As shown in Table 1, the current model for the VSOP-2 spacecraft has a $10 \mathrm{~m}$-class antenna in a slightly higher orbit than HALCA, operating at 5 (or possibly 8), 22 and $43 \mathrm{GHz}$. This model has 25 micro-arcsecond resolution at $43 \mathrm{GHz}$, and a sensitivity increase over the VSOP mission of one order of magnitude, allowing a wider scientific area and a larger number of observable sources.

The ARISE mission, to be proposed to NASA, is considering the use of a $25 \mathrm{~m}$ inflatable antenna for observations up to $86 \mathrm{GHz}$ (Ulvestad and Linfield 1998; Ulvestad 2000; Marscher 2000; see also http://arise.jpl.nasa.gov). ARISE was favorably mentioned in the National Research Council's decadal review "Astronomy and Astrophysics in the New Millennium" earlier this year, however the development of the necessary technologies to meet the science goals will probably result in a launch sometime after 2011.

The angular resolution with a HALCA-type orbit at $43 \mathrm{GHz}$ and $86 \mathrm{GHz}$ is 20 and 10 micro-arcseconds, respectively. This is only 10 and 5 times that of the suspected Schwarzschild radius in the case of M87. ASCA's discovery of MCG-6-30-15 type phenomena seen by Fe-line spectroscopy seems to be taking place in a region only several times that of the event horizon (Tanaka et al. 1995). Space VLBI in the future will see the region with comparable physical resolution with real imaging capability. Detailed three-dimensional magnetohydrodynamic simulations of the region surrounding the rotating supermassive black hole are now being carried out (e.g., Shibata 2000; Meier and Koide 2000), and these simulation results will be compared with the observational results of future missions.

The techniques required to realize future space VLBI missions are: development of large, accurate, deployable radioastronomy antennas; receiver cryogenics; accurate orbit determination; high bit-rate communications etc. Space VLBI is a most promising field both in terms of science and engineering capability.

Acknowledgments. The VSOP Project is led by the Japanese Institute of Space and Astronautical Science, with significant contributions from National Astronomical Observatory of Japan, the Jet Propulsion Laboratory, the U.S. National Radio Astronomy Observatory, the Canadian Dominion Radio Astrophysical Observatory, the Australia Telescope National Facility, the European VLBI Network, the Joint Institute for VLBI in Europe, and the directors and staff of many of the world's radio observatories. The author, who served as both VSOP Project Scientist and VISC co-chair, would like to express his sincere thanks to a large number of international members of the VSOP team for the collaborations on the VSOP for long years. The operational model for the VSOP mission has been developed in the wide radio-astronomy community, and it will form the basis, with refinements, for future missions. Philip Edwards is thanked for his help in the preparation of this paper.

\section{References}

Bolton, J.G. \& Stanley, G.J. 1948, Nature, 161, 312

Burke B.F. 1984, in VLBI and Compact Radio Sources, ed R. Fanti, K. Kellermann, G. Setti, IAU Symp. 110, 397 
Hirabayashi, H. et al. 1998, Science. 281, 1825

Hirabayashi, H., Hirosawa, H. et al. 2000a, PASJ in press

Hirabayashi, H., Fomalont, E.B. et al. 2000b, PASJ in press

Hirabayashi, H. et al. 2000c, in Astrophysical Phenomena Revealed by Space VLBI, ed. H. Hirabayashi, P. G. Edwards \& D. W. Murphy, (Sagamihara: ISAS) 277

Hirabayashi, H. et al. 2001, these proceedings

Jones, D.L. et al. 2000, in Astrophysical Phenomena Revealed by Space VLBI, ed. H. Hirabayashi, P. G. Edwards \& D. W. Murphy, (Sagamihara: ISAS) 265

Kardashev, N.S. 1997, Exp. Astron. 7, 329

Kardashev N.S., Slysh V.I. 1988, in The Impact of VLBI on Astrophysics and Geophysics, ed M.J. Reid, J.M. Moran, IAU Symp. 129, 433

Kobayashi, H., Shimoikura, T. et al. 2000, in Astrophysical Phenomena Revealed by Space VLBI, ed. H. Hirabayashi, P. G. Edwards \& D. W. Murphy, (Sagamihara: ISAS) 109

Levy G.S., Linfield R.P., Ulvestad J.S., Edwards C.D., Jordan F.J., DiNardo S.J., Christensen C.S., Preston R.A. et al. 1986, Science 234, 187

Linfield R.P., Levy G.S., Ulvestad J.S., Edwards C.D., DiNardo S.J., Stavert L.R., Ottenhoff C.H., Whitney A.R. et al. 1989, ApJ 336, 1105

Linfield R.P., Levy G.S., Edwards C.D., Ulvestad J.S., Ottenhoff C.H., Hirabayashi H., Morimoto M., Inoue M. et al. 1990, ApJ 358, 350

Marscher, A.P. 2000, in Astrophysical Phenomena Revealed by Space VLBI, ed. H. Hirabayashi, P. G. Edwards \& D. W. Murphy, (Sagamihara: ISAS) 261

Meier, D.L. \& Koide, S. 2000, in Astrophysical Phenomena Revealed by Space VLBI, ed. H. Hirabayashi, P. G. Edwards \& D. W. Murphy, (Sagamihara: ISAS) 31

Murata, Y., Hirabayashi, H. \& Edwards, P.G. 2001, these proceedings

Pilbratt G. 1991, in Radio Interferometry: Theory, Techniques, and Applications, ed T.J. Cornwell, R.A. Perley, ASP Conf. Ser. 19, 102

Schilizzi R.T. 1988, in The Impact of VLBI on Astrophysics and Geophysics, ed M.J. Reid, J.M. Moran, IAU Symp. 129, 441

Shibata, K. 2000, in Astrophysical Phenomena Revealed by Space VLBI, ed. H. Hirabayashi, P. G. Edwards \& D. W. Murphy, (Sagamihara: ISAS) 29

Tanaka, Y., Nandra, K., Fabian, A.C. et al. 1995, Nature, 375, 659

Ulvestad, J.S. 2000, Adv. Space Res. 26, 735

Ulvestad, J.S. \& Linfield, R.P. 1998, IAU Colloquium 164, ASP Conf. Series, 144,397 